\title{
Comparison of clinical and histopathological findings in patients with lupus nephritis having IgG deposits versus patients without IgG deposits in renal biopsies
}

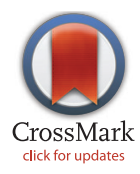

\author{
Hina Tariq ${ }^{1 *}$, Humaira Nasir ${ }^{1}$, Aniqa Khalid Kazi ${ }^{2}$, Alishba Khan ${ }^{2}$ \\ ${ }^{1}$ Histopathology Department, Shifa International Hospital, Islamabad, Pakistan \\ ${ }^{2}$ Shifa College of Medicine, Islamabad, Pakistan
}

\section{A R T I C L E I N F O}

Article Type:

Original

\section{Article History:}

Received: 8 July 2017

Accepted: 15 November 2017

Published online: 26 November 2017

\section{Keywords:}

Histopathological findings

IgG deposits

Lupus nephritis

Renal biopsy

\begin{abstract}
A B S T RAC T
Introduction: Up to $10 \%$ of lupus nephritis (LN) do not show IgG deposition of immunofluorescence (IF). Our rationale is to find out whether there is any significant difference in clinical presentation and histopathological findings in both groups of patients. Objectives: To compare clinical and histopathological findings in patients with LN having IgG deposits versus patients without IgG deposits in renal biopsies.

Patients and Methods: Two groups of IgG positive and IgG negative were made and their details were recorded. Data were analyzed by SPSS 20. Fischer's exact test was applied to determine significance of above mentioned clinical and laboratory findings with/without IgG deposits in renal biopsies.

Results: Fischer's exact test results' for all variables was as follows; M: F ratio was 0.5 , edema 0.10 , deranged RFT 0.3 , hematuria 0.10 , proteinuria 0.4 , anti-double stranded DNA antibody 0.3 and anti-nuclear antibody 0.5 . The $P$ values of LN were class II 0.003 , class III 0.8 and class IV 0.3. These findings show that groups with no IgG deposits on IF, present with milder clinical disease.

Conclusion: There was a marginal significant $P$ value for hematuria, edema and LN class II, pointing towards the fact that relatively milder disease in IgG negative group as compared to IgG positive group.
\end{abstract}

Implication for health policy/practice/research/medical education:

Article has pointed towards the fact that even in small percentage of cases without IgG deposits on immunofluorescence, clinical and histopathological findings have no significant difference from cases with IgG deposits.

Please cite this paper as: Tariq H, Nasir H, Kazi AK, Khan A. Comparison of clinical and histopathological findings in patients with lupus nephritis having IgG deposits versus patients without IgG deposits in renal biopsies. J Renal Inj Prev. 2018;7(1):22-26. DOI: 10.15171 /jrip.2018.05.

\section{Introduction}

Systemic lupus erythematosus (SLE) is one of the most common autoimmune disorders in women during their reproductive age. The female to male ratio is 9:1 (1). Renal disease develops in more than half of SLE patients, and represents the first clinical manifestation of SLE in 15\%$20 \%$ (2). Male patients had a more severe lupus nephritis (LN) as compared to women (3).

$\mathrm{LN}$ is an important reason for morbidity and even mortality in patients with SLE. It has multiple morphologic manifestations with various clinical presentations and long-term consequences. The pathogenesis primarily involves immune complexes (2).

When patients with SLE present with urinary abnormalities, a renal biopsy is usually needed to rule-out or confirm LN (4). A renal biopsy examined by routine light microscopy along with immunofluorescence (IF) contributes towards diagnosis, providing prognostic information and aids in appropriate management $(2,5)$. Histological, ISN/RPS 2003 has described six different classes based on the morphologic lesions, extent and severity of the involvement, immune complex deposition, 
activity and chronicity of lesions (5). There are different treatment strategies, for each of the class of LN (6). Most of the patients suffering from SLE have renal abnormalities, if histopathology is supplemented by IF (1). Treatment and prognosis range from excellent with only observation in a case with minimal mesangial deposits, to renal failure despite aggressive immunosuppression in patients with severe proliferative disease (2).

In LN, immune deposits can be detected in all renal compartments, including glomeruli, tubules, interstitial area and blood vessels. The pattern of IF is extremely helpful in confirming the diagnosis of LN when the diagnosis of SLE is doubtful at the time of biopsy. IgG is found almost universally, while co-deposition of IgM and $\operatorname{IgA}$ is present in most specimens. The combined deposition of the three immunoglobulins (Igs) with C3 and $\mathrm{Clq}$ is designated as a full-house pattern, which is a characteristic finding of $\mathrm{LN}$ and uncommon in other renal diseases. The complement 3 (C3) is the most frequent complement which stains intensely, followed by C1q (7). Up to $10 \%$ of LN do not show IgG deposition of IF (8). After an extensive literature search, nothing regarding documentation of any differences in IgG positive and IgG negative groups in the context of clinical, laboratory and histopathological patterns was found. Our rationale is to find out whether there is any significant difference exists in clinical presentation and histopathological findings in both groups of patients.

\section{Objectives}

To compare clinical and histopathological findings in patients with LN having IgG deposits versus patients without IgG deposits in renal biopsies.

\section{Patients and Methods}

\section{Study and setting}

This is a retrospective study, conducted at histopathology department, Shifa International Hospital, Islamabad, from January 2008 to June 2014.

\section{Definition of lupus nephritis}

Patients who were already diagnosed as SLE based on last published American College of Rheumatology guidelines and had renal biopsies were included.

\section{Clinical and laboratory data}

Non-probability, consecutive sampling was applied. Cases were retrieved from laboratory system, in which two cores were received, one for routine microscopy and other for IF for polyclonal rabbit antihuman IgG, IgA, IgM, C3 and $\mathrm{C} 1 \mathrm{q}$ conjugates were included. The assessment of biopsies was performed according to ISN/RSP 2003 classification (9).

\section{Ethical issues}

The research followed the tenets of the Declaration of Helsinki. The research was approved by the ethical committee of Shifa International Hospital, Islamabad, Pakistan. All patients' information remained confidential.

\section{Statistical analysis}

Two groups of IgG positive and IgG negative were established and their details, including edema, hematuria, nephrotic range proteinuria, sub-nephrotic range proteinuria, as well as renal function test (RFT), antidsDNA, ANA and LN classes as per ISN/RSP were noted. Data were analyzed in statistical software SPSS 20. Frequency and percentage of qualitative variables like gender, edema, hematuria, nephrotic range proteinuria, sub-nephrotic range proteinuria, deranged RFT and LN classes were calculated. Mean of age was calculated for males and females separately. Fischer's exact test was applied to determine the significance of above mentioned clinical, laboratory and histopathological findings with/ without IgG deposits in renal biopsies on IF.

\section{Results}

A total of 92 renal biopsies, including 76 females and 16 males patients were included in the study (Figure 1). The mean age of presentation was 26 years for females and 25 years for males. Among female patients, 69 had IgG deposits on IF and 7 had no IgG deposits. All 16 males had IgG deposits on IF, hence, no comparison could be made (Table 1).

In IgG positive group, majority of cases fall in LN class IV, followed by class V. Class III and II had equal distribution. Only 2 cases were found to be class VI while only one case was of class I LN. In IgG negative group, three cases were of LN class II, while two cases were of LN classes III and IV each (Table 2).

The results of clinical and laboratory variables, including edema, hematuria, proteinuria, deranged RFT, antidsDNA and ANA, are summarized in Table 2.

All above mentioned clinical and laboratory variables were compared with histopathological classes of LN by Fischer's exact test. Results for all variables was as follows; M: F 0.5, edema 0.10 , deranged RFT 0.3 , hematuria 0.10 , proteinuria 0.4 , anti-double stranded DNA antibody 0.3 and anti-nuclear antibody 0.5 . The $\mathrm{P}$ values of $\mathrm{LN}$ were class II; 0.003 , class III; 0.8 and class IV; 0.3 . Out of all

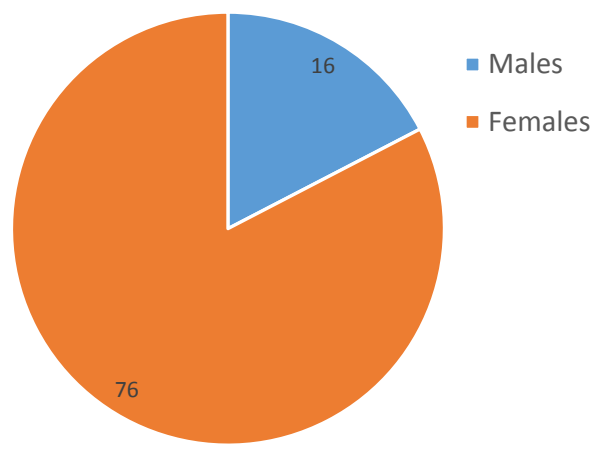

Figure 1. Gender distribution. 
these, only edema, hematuria and LN class II were found to be marginal significant $P$ value. (Table 1) These findings showed that groups with no IgG deposits on IF, present with milder clinical disease.

\section{Discussion}

The SLE is a multisystem autoimmune disease. Different antibodies are detected in sera of different sets of patients among which most common are ANA and anti-dsDNA antibodies. Diagnosis of LN cannot be based on solely based on renal biopsy. Clinicopathological correlation is necessary for the definitive diagnosis (9).

In the literature, it has been documented that in LN by direct immunofluorescent technique, out of all antibodies and complements, IgG deposition is found positive in more than $90 \%$ of cases. While $\operatorname{IgA}$ and $\operatorname{IgM}$ staining are observed in $60 \%-70 \%$ of cases and C3, C1q make up around $80 \%$ of $\mathrm{LN}$ cases (8).

LN classes have different clinical presentations. Most of the patients who are symptomatic usually have an advanced class. The RFT and urine results are normal in class I. Hence these patients are usually not biopsied and therefore true incidence is not known (10).

The LN class II is mostly found to have microscopic hematuria or non-nephrotic range proteinuria and normal RFT. Proteinuria, which is occasionally nephrotic range along with hematuria and deranged RFT are present in class III. LN class IV has severe disease and mostly are biopsied for hematuria, proteinuria, nephrotic syndrome, renal failure and hypertension. Nephrotic range proteinuria is the most common mode of presentation of class V. Class VI is a clinical diagnosis, but is suspected on renal biopsy, if more than $90 \%$ of total glomeruli are globally sclerosed. These patients have deranged renal function in conjunction with proteinuria and normal urinary sediment (10).

ANA is positive in the majority of cases of $\mathrm{LN}$, but antidsDNA titers are low or even absent in more than $50 \%$ of the cases (10). Comparison of ANA along with other findings of our study with other studies were tabulated in Table 2.

Pattern and intensity of immunofluorescence vary with LN class. Renal biopsy is unremarkable in class I of LN (9). The classes I and II have mesangial deposits of Igs and complements. In class III, mesangial and capillary wall deposits are noticed in a segmental distribution. There are intense, global and diffuse sub-endothelial deposits along with mesangial deposits in class IV (10).

Table 1. Comparison of clinical, laboratory and histopathological findings in IgG positive and IgG negative lupus nephritis patients

\begin{tabular}{|c|c|c|c|}
\hline $\begin{array}{l}\text { Clinical, laboratory and histopathological } \\
\text { findings }\end{array}$ & $\begin{array}{l}\text { IgG positive cases } \\
\text { No. (\%) }\end{array}$ & $\begin{array}{l}\text { IgG negative cases } \\
\text { No. (\%) }\end{array}$ & Fischer's exact test \\
\hline Male & $16(17.4)$ & - & - \\
\hline Female & $69(75.0)$ & $7(7.6)$ & - \\
\hline Male: female ratio & - & - & 0.5 \\
\hline Edema & $57(67.0)$ & $3(42.8)$ & 0.10 \\
\hline Hematuria & $27(32.0)$ & $1(14.3)$ & 0.10 \\
\hline Proteinuria & $45(53.0)$ & $2(28.6)$ & 0.4 \\
\hline Deranged RFT & $25(29.4)$ & $1(14.3)$ & 0.3 \\
\hline Anti-dsDNA & $23(27.0)$ & $4(57.1)$ & 0.3 \\
\hline ANA & $43(50.6)$ & $3(42.8)$ & 0.5 \\
\hline \multicolumn{4}{|l|}{ Lupus nephritis classes } \\
\hline Class I & $1(1.2)$ & 0 & - \\
\hline Class II & $10(11.8)$ & $3(42.8)$ & 0.003 \\
\hline Class III & $10(11.8)$ & $2(28.6)$ & 0.8 \\
\hline Class IV & $43(50.6)$ & $2(28.6)$ & 0.2 \\
\hline Class V & $19(22.3)$ & 0 & 0 \\
\hline Class VI & 0 & - & - \\
\hline
\end{tabular}

Table 2. Frequencies and percentages of selected study variables in our study and other studies

\begin{tabular}{|c|c|c|c|c|c|c|c|}
\hline & $\begin{array}{c}\text { Hematuria } \\
\text { No. (\%) }\end{array}$ & $\begin{array}{c}\text { Proteinuria } \\
\text { No. (\%) }\end{array}$ & $\begin{array}{c}\text { Deranged RFT } \\
\text { No. (\%) }\end{array}$ & $\begin{array}{c}\text { ANA } \\
\text { No. (\%) }\end{array}$ & $\begin{array}{c}\text { Anti-ds DNA } \\
\text { No. (\%) }\end{array}$ & $\begin{array}{c}\text { IgG positive } \\
\text { No. (\%) }\end{array}$ & $\begin{array}{c}\text { IgG negative } \\
\text { No. (\%) }\end{array}$ \\
\hline Our study ( $n=92$ ) & $28(30.4)$ & $47(51.0)$ & $26(28.3)$ & $46(50.0)$ & $27(29.3)$ & $85(92.4)$ & $7(7.6)$ \\
\hline Iraq $2013(n=2)$ & $2(100)$ & $2(100)$ & $2(100)$ & $2(100)$ & $2(100)$ & $2(100)$ & - \\
\hline Sydney $2013(n=71)$ & $18(25.3)$ & $40(56.3)$ & - & - & - & $66(93)^{*}$ & - \\
\hline Brazil $2013(n=11)$ & - & - & - & - & - & $11(100)$ & - \\
\hline Kashmir $2012(n=35)$ & - & - & - & $35(100)$ & $20(57.14)$ & $32(91)$ & $3(9)$ \\
\hline Thailand $2015(n=244)$ & $157(64.3)$ & $125(51.2)$ & - & - & 190 (77.9) & - & - \\
\hline Saudi Arabia 2014 ( $n=148)$ & - & 105 (89.7) & $47(34.8)$ & $112(95.7)$ & $112(95.7)$ & $123(98.4)$ & $2(1.6)$ \\
\hline New York $2002(n=80)$ & - & - & - & - & - & $80(100)$ & - \\
\hline
\end{tabular}


In LN class $\mathrm{V}$, there are mostly diffuse sub-epithelial granular deposits of IgG and C3, accompanied by C1q. In class IV, occasional pauci-immune cases are observed, usually in a subclass of LN IV-S (sclerosing/chronic lesions present (10).

The IgG has been recognized to be the most important antibody regarding the pathogenesis of LN. Human IgG molecules have been classified into four different IgG subclasses based on difference in constant region of the heavy chain (10).

IgG is most abundant (10\%-20\%) of all immunoglobulins in human serum. It has four subclasses and is named according to their distribution, IgG1, IgG2, IgG3, and IgG4. These subclasses have different functions. IgG1 is induced by antigens and is the most abundant subclass. IgG2 responses to bacteria but has poor response, primarily due to its short half-life. Following repeated or longterm exposure to a particular antigen, IgG4 antibodies are formed. IgG is documented to show marked genetic variation and its expected impact beyond the serological findings and even missed by current techniques. IgG1 and IgG3 are known to play a central role in immune mechanisms, whereas IgG2 and IgG4 induce weak responses. Different allotypes in IgG of a given individual can be the reason of varied clinical presentations among individuals and races (10).

LN class $\mathrm{V}$ accounts for approximately $10 \%$ of all renal lesions of SLE. Peculiar point about this class is that these patients may have a clinical presentation similar to idiopathic membranous nephropathy $(\mathrm{MN})$. This class does not show a 'full-house' pattern. Hass (11) evaluated 14 cases of LN class V and found out that only nine cases exhibited 'full-house' pattern. In 1992, two years before Haas, Iskandar et al reported that IgG3 dominated in most cases of diffuse proliferative LNs, in contrast to idiopathic $\mathrm{MN}$, in which IgG4 is predominant. Thus pointing to the fact that antibodies of IgG subclasses might be helpful in difficult cases.

In 2002, Kuroki et al found no significant difference in the deposition of various subclasses of IgG in class IV of LN. However, the mean score of each IgG3 and IgG4 deposition was higher than those of IgG2 (9).

Clinical presentation and histopathological findings also influence immune complexes deposition, regarding their location, quantity, as well as their capability to elicit an inflammatory response. It has been documented that overall deposition of glomerular IgG correlates with the severity of LN. In 2003, Zuniga et al documented that IgG2 and IgG3 were the predominant subclasses of IgG in LN classes (12).

In 2014, Yin et al analyzed different patterns of renal IF staining for IgG subclasses in idiopathic $\mathrm{MN}$ and class V LN, in which $14 \%$ were diagnosed as class V of LN. They proposed that difference in intensity patterns between IgG4 and IgG3 and intensity of C1q could serve as a diagnostic tool in differentiating class $\mathrm{V}$ of $\mathrm{LN}$ from idiopathic MN (13).

Some other investigators proposed that IgA nephropathy (IgAN) may be a special clinical subtype of SLE. Up to 15 cases are documented in the literature, including 5 cases of Hongyan et al. They had documented 5 atypical cases of LN, which showed predominant deposition of IgA. All of the five cases had multiple-system involvements including kidneys. Two cases had gross hematuria, 3 had microscopic hematuria; all 5 had proteinuria, 2 had renal dysfunction while 2 presented with edema. All were positive for ANA while 3 were positive for anti-dsDNA (14).

Atypical LN differs from classical IgA nephropathy, which mainly presents in men during their second and third decades of life. They usually have a previous history of respiratory or gastrointestinal infection. Clinical presentations are gross or microscopic hematuria, edema, and hypertension and also deranged RFT. While atypical LN presents in older age compared to IgA nephropathy, and mostly in women. These patients have no prior history of infection or raised IgA levels. One of these cases had C3 deposit on IF while other four cases had IgM and C3 deposits (14). Two of our cases belong to this atypical category of LN. One of these cases was a 20-year-old female patient, who had ANA and anti-dsDNA. She had class II LN, with equivocal staining for IgG and moderate mesangial deposits for IgA. This might represent an atypical LN. Another case was 20 -year female, with DNA positivity and nephrotic range proteinuria. She had class II LN, with negative staining for IgG and mild mesangial deposits for IgA. Rests of the cases were equivocal positive for IgG and IgA.

Nisihara et al had positive IgG, C3, and C1q in all study cases. Seventy-three percent showed deposition of IgM and $45 \%$ had IgA deposition in glomeruli. The "fullhouse" pattern was observed in $36 \%$ patients. Major limitation of this study was small number of cases (5). In our cases, $92.5 \%$ had IgG deposits, 78.5\% had IgA, 82\% had IgM, 96.8\% had C3 and $67.7 \%$ had C1q. Full-house pattern was documented in $54.8 \%$ of total cases.

Lack of IgG deposition may be the result of differences of subclasses distribution in different classes of LN or due to numerous allotypes of IgG subclasses in different ethnic groups. Reason of no IgG deposits needs to be further exploited along with whether its absence really affects clinical presentation and long-term prognosis of patients.

\section{Conclusion}

In our study, a marginal significant p-value for hematuria, edema and LN class II was seen, in IgG negative group as compared to IgG positive group. These findings pointing towards the fact regarding a relatively milder disease in IgG negative group as compared to IgG positive group. However, there is no significant difference in other variables of the study. Further studies are needed, to find a true difference in the pattern of clinical presentation and histopathological findings, regarding IgG deposition on 
immunofluorescence.

\section{Limitations of the study}

Currently no literature could be found in this context. Hence further research needs to be carried out to know a true weight age in the clinical context.

\section{Authors' contribution}

$\mathrm{HN}$ conceived the idea, interpreted results of light and immunofluorescent microscopy. AKK and AK collected data. HT analyzed data and prepared manuscript. All authors read, revised and approved the final manuscript.

\section{Conflicts of interest}

The authors declared no competing interests.

\section{Ethical considerations}

Ethical issues (including plagiarism, misconduct, data fabrication, falsification, double publication or submission, redundancy) have been completely observed by the authors.

\section{Funding/Support}

None.

\section{References}

1. Anders HJ, Fogo AB. Immunopathology of lupus nephritis. Semin Immunopathol. 2014;36:443-59. doi: 10.1007/ s00281-013-0413-5.

2. Seshan SV, Jennette JC. Renal disease in systemic lupus erythematosus with emphasis on classification of lupus glomerulonephritis: advances and implications: Arch Pathol Lab Med. 2009;133:233-48. doi: 10.1043/1543-2165133.2.233.

3. Haubitz M. [Lupus nephritis]. Z Rheumatol. 2012;71:87584. doi: $10.1007 / \mathrm{s} 00393-012-1105-6$

4. D'Agati VD, Stokes MB. Renal Disease in Systemic Lupus Erythematosus, Mixed Connective Tissue Disease, Sjogren's Syndrome, and Rheumatoid Arthritis. In: Jennette JC, Olson JL, Schwartz MM, Silva FG, eds. Heptinstall's Pathology of the Kidney. 6th ed. Vol 1. Philadelphia: Wolters Kluwer/
Lippincott Williams \& Wilkins; 2007

5. Nisihara RM, Magrini F, Mocelin V, Messias-Reason IJ. Deposition of the lectin pathway of complement in renal biopsies of lupus nephritis patients. Hum Immunol. 2013;74:907-10. doi: 10.1016/j.humimm.2013.04.030.

6. Weening JJ, D’Agati VD, Schwartz MM, Seshan SV, Alpers CE, Appel GB, et al. The classification of glomerulonephritis in systemic lupus erythematosus revisited. J Am Soc Nephrol. 2004;15:241-50.

7. Yong JL, Killingsworth MC, Lai K. Renal biopsy pathology in a cohort of patients from southwest Sydney with clinically diagnosed systemic lupus erythematosus. Int J Nephrol Renovasc Dis. 2013;6:15-26. doi: 10.2147/IJNRD.S34357.

8. Seshan SV, Jennette JC. Renal disease in systemic lupus erythematosus with emphasis on classification of lupus glomerulonephritis: advances and implications. Arch Pathol Lab Med. 2009;133:233-48. doi: 10.1043/1543-2165133.2.233.

9. Kuroki A, Shibata T, Honda H, Totsuka D, Kobayashi K, Sugisaki T. Glomerular and serum igg subclasses in diffuse proliferative lupus nephritis, membranous lupus nephritis, and idiopathic membranous nephropathy. Intern Med. 2002;41:936-42.

10. Jefferis R, Lefranc MP. Human immunoglobulin allotypes: possible implications for immunogenicity. MAbs. 2009;1:332-8.

11. Haas M. IgG subclass deposits in glomeruli of lupus and nonlupus membranous nephropathies. Am J Kidney Dis. 1994;23:358-64.

12. Zuniga R, Markowitz GS, Arkachaisri T, Imperatore EA, D’Agati VD, Salmon JE: Identification of IgG subclasses and C-reactive protein in lupus nephritis: The relationship between the composition of immune deposits and Fc gamma receptor type IIA alleles. Arthritis Rheum 2003; 48:460-70. doi: 10.1002/art.10930.

13. Yin G, Hang L, Na L, Chao L, Lin D, Yan L, et al. Different patterns of renal immunofluorescence staining for IgG subclasses in idiopathic and secondary membranous nephropathy. Chinese J Nephrol. 2014;30:85-91.

14. Hongyan L, Yi Z, Bao D, Yuewu L, Juan M. A study on clinical and pathologic features in lupus nephritis with mainly IgA deposits and a literature review. Clin Dev Immunol. 2013;2013:289316.

Copyright ( $) 2018$ The Author(s); Published by Nickan Research Institute. This is an open-access article distributed under the terms of the Creative Commons Attribution License (http://creativecommons.org/licenses/by/4.0), which permits unrestricted use, distribution, and reproduction in any medium, provided the original work is properly cited. 